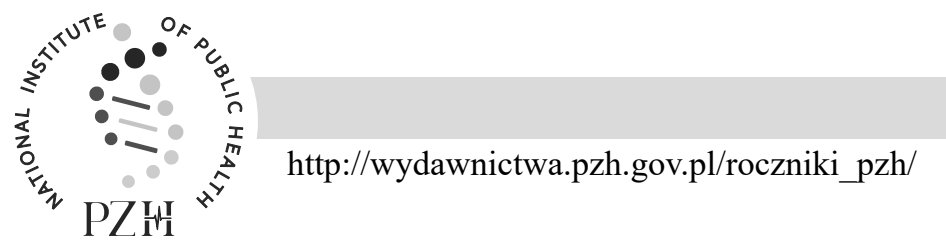

\title{
EMERGENCY MANAGEMENT OF DENTAL INJURY; PREPAREDNESS AMONG SCHOOL TEACHERS IN BHUBANESWAR, INDIA
}

\author{
Ramesh Nagarajappa ${ }^{1}$, Debasruti Naik ${ }^{l}$ \\ ${ }^{1}$ Department of Public Health Dentistry, Institute of Dental Sciences, Siksha 'O' Anusandhan \\ (Deemed to be University), Bhubaneswar, Odisha, India
}

\begin{abstract}
Background. School is the place, identified with a noticeable risk of Traumatic Dental Injuries (TDI) in children which have functional, esthetic and psychological effects.

Objectives. To assess the preparedness of concerning traumatic dental injuries and their management among school teachers and also to empower the clinician to frame a set of instructions for school teachers to handle the emergencies effectively at the site of the incident.

Material and Methods. A cross-sectional study was conducted among 330 school teachers enrolled in government and private schools of 24 randomly selected schools in Bhubaneswar city. A self-administered questionnaire was distributed to collect information on participants demographic characteristics, knowledge, attitude and practice about emergency management of Traumatic Dental Injury. Chi-square test with level of significance set at 5\% was used for statistical analysis.

Results. Statistically significant $(\mathrm{p}<0.05$ ) correct responses were provided by $66.7 \%$ males and $35.6 \%$ females and $100 \%$ younger age teachers. Larger population perceived that their level of knowledge was not satisfactory as they responded for inadequate and don't know options which was significant in relation to gender and age $(\mathrm{p}<0.05)$ but not with respect to the type of school $(\mathrm{p}>0.05)$. All the respondents expressed the need for a training program.

Conclusions. This study highlights the instantaneous need for tailor made dental health educational and preventive programs for school teachers in order to effectively manage the Traumatic Dental Injuries.
\end{abstract}

Key words: knowledge, management, school teachers, Traumatic Dental Injury

\section{INTRODUCTION}

Childhood is a social construction; whoseboundaries shift with time and place and this has implications for vulnerability to injury. Epidemiological studies have shown that prevalence of Traumatic Dental Injury (TDI) ranges between $11 \%$ to $30 \%$ and $4 \%$ to $58 \%$ in primary and permanent dentition respectively [13, 28]. Luxation injuries are the most common TDIs in primary dentition, whereas crown fractures are more commonly reported for permanent teeth. This variability may have various causes, including the type of study, trauma classification, strategy, study size and population, geographical area, and contrasts in cultural behavior [13].

Most dental injuries result from simple falls, accidents, sports activities, or childish pranks, which were not intended to cause harm [3, 13, 28]. Children with TDIs can experience emotional stress, teasing, and distress, influencing their self-image both at the time of mishap, as well as later during treatment [1, 21]. Moreover, there is a relationship between dental issues and scholastic accomplishment and learning in children [22].

Dental trauma is one of the most important oral health problems in children and is considered a dental public health problem because: (a) incidence of TDI is high world-wide; (b) trauma to oro-facial structures can cause severe damage to teeth, supporting structures, and craniofacial structures; (c) oro facial injuries can occur at a young age, and treatment may continue for rest of the patient's life.

Children spend large span of time in school where accident from falls is very common and is the main etiological factor of TDI at school [3, 28]. School teachers are the first ones contacted by the child after accident. Hence, they are expected to have proper knowledge and awareness, which will help them

Corresponding author: Debasruti Naik, (ORCID ID: 0000-0003-2476-3451), Department of Public Health Dentistry, Institute of Dental Sciences, Siksha 'O' Anusandhan (Deemed to be University), Bhubaneswar - 751003, Odisha, India, Mobile: +919439238604, e-mail: smilewithdebshruti@gmail.com 
to carry out expedite procedures in time. Further, proper diagnosis, treatment planning and follow-up assures a favourable outcome. Therefore, the present investigation was set out with the following objectives: (1) to assess the knowledge, attitude and practice towards the emergency management of dental trauma among school teachers of Bhubaneswar (India) and (2) to enable the clinician to formulate a set of instructions which can be given to school teachers, such that the emergencies can be handled effectively.

\section{MATERIAL AND METHODS}

\section{Study design and ethical approval}

This cross-sectional descriptive survey was steered from July to August 2018 among 330 primary and secondary school teachers of Bhubaneswar, India. School teachers who were permanent residents of Bhubaneswar city, aged over 18 years and were logically and physically proficient of responding to questionnaire in English language were included in the study. Teachers who did not give their consent to participate and those do not present on the day of survey were excluded.

The survey procedure was approved by Institutional Ethical Committee (Ref No/DMR/ IMS.SH/SOA/180074). Prior to initiation of primary investigation, certified list of all schools (Government and Private) of Bhubaneswar city was attained from District Education Office (DEO), Bhubaneswar. An official authorization was attained from corresponding Heads of designated schools. Filling out the questionnaire itself was considered consent by participating teachers.

\section{Compilation of study subjects}

School teachers were enrolled by a two-stage stratified random sampling technique. In the primary stage, Bhubaneswar city was alienated into four geographical areas, and six schools (two Government and four private) from each area were randomly chosen. Of the total number of Government (163) and private schools (319), eight Government and 16 private schools were randomly picked. In the subsequent stage, qualified school teachers were stratified by age and gender and randomly selected in proportion to the aggregate number of teachers accessible in each school which accomplished for a sample of 330 .

\section{Pre-testing survey}

A self-administered structured questionnaire was developed in English and pretested on a group of 15 school teachers. Face validity specifies whether the instrument seems to be evaluating the anticipated qualities. When face validity was assessed, it was detected that $96 \%$ of participants found the questionnaire to be easy. Assessment of content validity reproduces a decision whether the instrument samples all the relevant or significant domains. Mean content validity ratio was intended as 0.89 based on the thoughts stated by a panel of four academicians. Test of reliability encompassed two components: Question-question reliability, which was calculated by percentage of agreement (88\%) and internal reliability for the responses to questions, which was evaluated using Cronbach's alpha coefficient (0.86).

\section{Questionnaire}

Ultimate questionnaire comprised of 36 questions under subsequent sections:

Section I: incorporated five questions to gather information related to teacher's demographic characteristics.

Section II: integrated 13 multiple choice questions to assess the knowledge in emergency management of TDI.

Section III: comprised of 12 questions, which aimed to assess the attitude of teachers toward the emergency management of TDI. Responses were recorded on a five-point Likert scale as "strongly agree", "agree", neither agree nor disagree", "disagree" and "strongly disagree".

Section IV: contained six multiple choice questions aimed to investigate the practice of teachers regarding emergency management of TDI.

\section{Data collection}

The nature and purpose of study were enlightened to the teachers. Its voluntary nature was accentuated, and confidentiality was guaranteed. Questionnaire was distributed to school teachers by a single investigator, offered adequate time to fill it and collected on the spot after completion. During this process the investigator was available to teachers for any clarifications with the questionnaire, simultaneously assured that the respondents answered sincerely without any access to other sources and also established that every question was answered.

\section{Statistical analysis}

The data collected was subjected for statistical analysis using SPSS version 21.0 (SPSS Inc., Chicago, Illinois, USA). For the purpose of analysis, proportion of KAP scores was used to assess the level of knowledge, attitude and practice among the participants. Correct responses of $<40 \%, 40-60 \%$, 
$60-80 \%$ and $>80 \%$ were categorized as having poor, average, good and very good KAP scores respectively. Descriptive statistics were used to summarize the data. $C h i$-square test $\left(\chi^{2}\right)$ was used to assess the relationship between categorical variables. For all tests, confidence interval and 'p-value' were fixed at $95 \%$ and $<0.05$ correspondingly.

\section{RESULTS}

A total of 330 school teachers, 60 (18.2\%) males and $270(81.8 \%)$ females consented and participated in the study. More than half, $175(53 \%)$ the study population were in the age group of 30 to 39 years and the mean age was $34 \pm 2.3$ years. A proportion of $81.8 \%$ and $18.2 \%$ represented the private and government schools respectively (Table 1).

Table 1. Demographic characteristics of study participants $(\mathrm{n}=330)$

\begin{tabular}{|l|c|}
\hline \multicolumn{1}{|c|}{ Characteristics } & $\mathrm{n}(\%)$ \\
\hline Gender: & \\
Male & $60(18.2)$ \\
Female & $270(81.8)$ \\
\hline Age Group (years): & \\
$20-29$ & $39(11.8)$ \\
$30-39$ & $175(53.0)$ \\
$40-49$ & $78(23.6)$ \\
$>50$ & $38(11.5)$ \\
\hline Type of school: & \\
Private & $270(81.8)$ \\
Government & $60(18.2)$ \\
\hline
\end{tabular}

Regarding knowledge on avulsion of tooth (Table 2), $66.7 \%$ males and $35.6 \%$ females and $100 \%$ younger age teachers provided the correct response which was statistically significant $(p<0.05)$. Contradictory responses were obtained for necessity of saving permanent tooth $(66.7 \% \mathrm{v} / \mathrm{s} 35.6 \%)$, right method of carrying the affected tooth to dentist $(66.7 \% \mathrm{v} / \mathrm{s} 14.8 \%)$. On the basis of school type, government teachers $(60 \%)$ gave higher correct responses but the difference was not statistically significant $(p>0.05)$. For all other knowledge-based questions, majority of males have given correct response which was statistically significant $(p<0.05)$. Responses on the basis of age has shown that a greater number of right answers were provided by younger age population. Government school teachers demonstrated better knowledge than their counterparts which was statistically significant $(p<0.05)$. Larger population perceived that their level of knowledge was not satisfactory as they responded for inadequate and don't know options which was significant in relation to gender and age $(p<0.05)$ but not with respect to type of school $(\mathrm{p}>0.05)$.

Male school teachers showed a significantly improved attitude than females $(\mathrm{p}<0.05)$. More of negative attitude was observed in elderly age group subjects compared with other groups. Responses categorized with reference to school type was almost alike in both private and government teachers which was statistically not significant $(p>0.05)$ (Table 3). A statistically significant $(\mathrm{p}<0.05)$ observation was found across all groups wherein they felt the emergency management and their timely intervention towards dental injuries was essential. Every participant expressed the need for receiving more information on proper management of traumatic dental injuries.

Practices followed by study population differed significantly between groups for all characteristics (Table 4). Additional healthier practices were reported in males than females for appropriate management of avulsed tooth. Across all age groups again a greater number of younger teachers were found to practice better methods which was statistically significant $(p<0.05)$. Similar findings were observed which did not differ much in both private and government school teachers. 


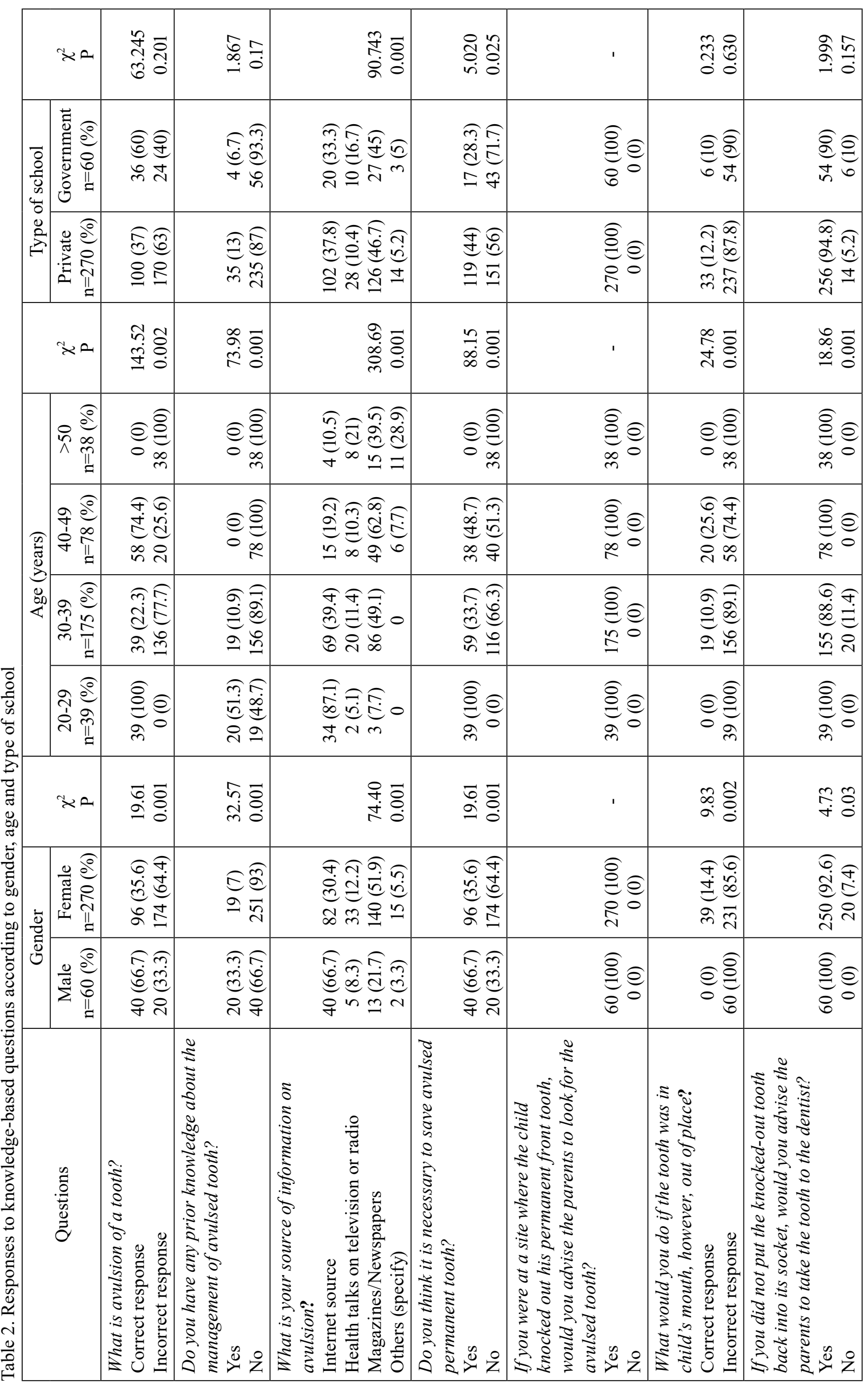




\begin{tabular}{|c|c|c|c|c|c|}
\hline $\begin{array}{l}\hat{o} \\
0 \\
0\end{array}$ & 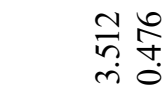 & $\begin{array}{l}\text { ते } \\
\text { ले } \\
\text { ले }\end{array}$ & 帒 & $\begin{array}{l}\tilde{\omega} \\
\infty \\
0 \\
\dot{\Xi} \\
\dot{\Xi}\end{array}$ & 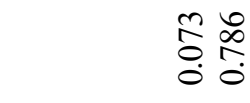 \\
\hline 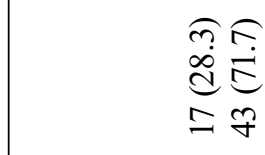 & 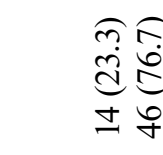 & 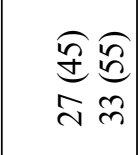 & 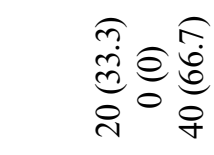 & 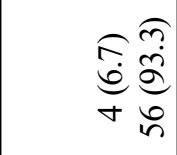 & 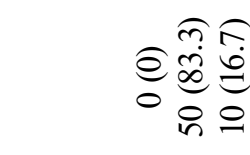 \\
\hline $\begin{array}{l}\widehat{\mathscr{C}} \overparen{\varrho} \\
\bar{\infty} \stackrel{\infty}{ }\end{array}$ & $\begin{array}{l}\stackrel{0}{f} \\
\stackrel{d}{d} \\
d \\
0 \\
0 \\
0\end{array}$ & 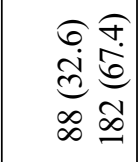 & 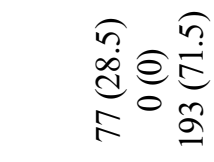 & 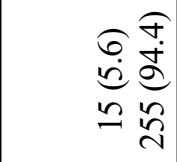 & 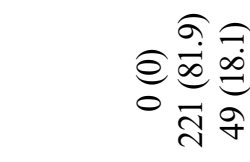 \\
\hline $\begin{array}{l}n \\
\infty \\
0 \\
\dot{0} \\
0\end{array}$ & 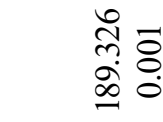 & 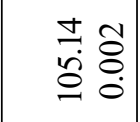 & 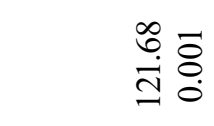 & 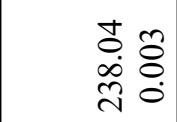 & $\begin{array}{l}\hat{b}: \overline{8} \\
\text { si: }\end{array}$ \\
\hline 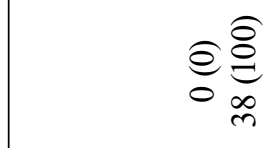 & 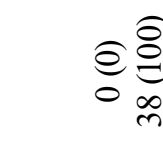 & 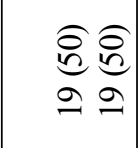 & 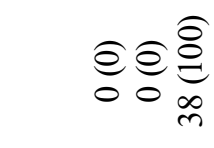 & 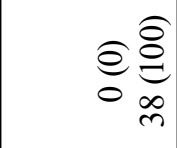 & 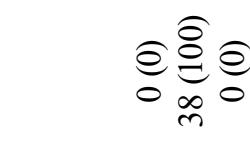 \\
\hline 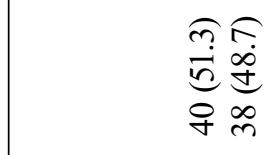 & 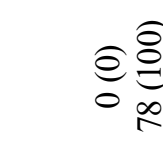 & 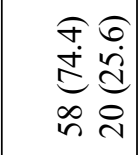 & 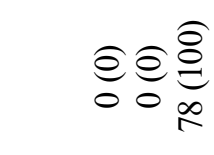 & 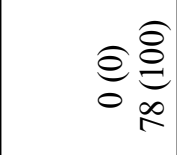 & 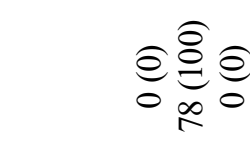 \\
\hline 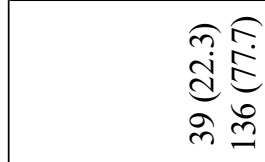 & 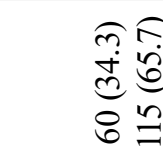 & 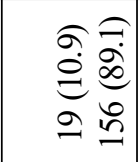 & 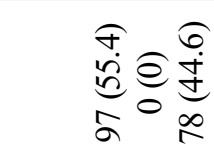 & 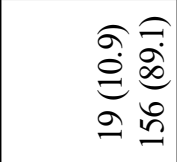 & 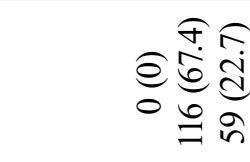 \\
\hline 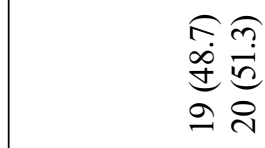 & 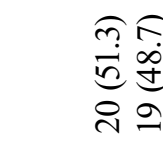 & 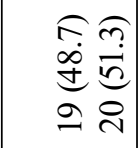 & 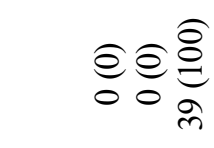 & 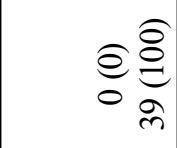 & 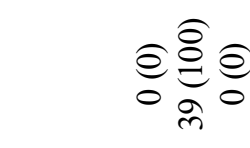 \\
\hline $\begin{array}{l}\stackrel{n}{\infty} \bar{g} \\
\stackrel{\Xi}{\Xi}\end{array}$ & 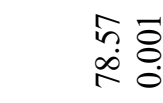 & $\begin{array}{l}\hat{N} \vec{b} \\
\text { ले } \\
\text { ले }\end{array}$ & 䓢 & $\begin{array}{l} \pm \delta \\
\vdots \\
i \\
0\end{array}$ & $\begin{array}{l}\text { @o } \\
=\end{array}$ \\
\hline 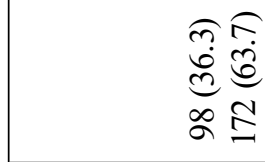 & 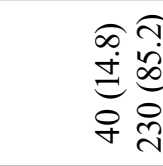 & 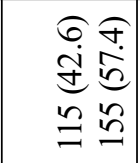 & 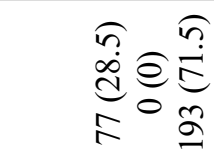 & $\begin{array}{l}\hat{E} \hat{\theta} \\
\hat{\sigma} \bar{n}\end{array}$ & 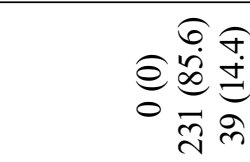 \\
\hline @e & 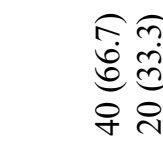 & 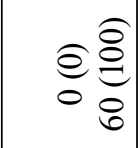 & 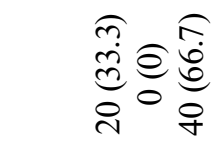 & 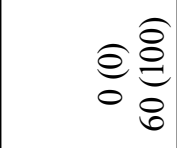 & 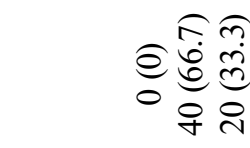 \\
\hline 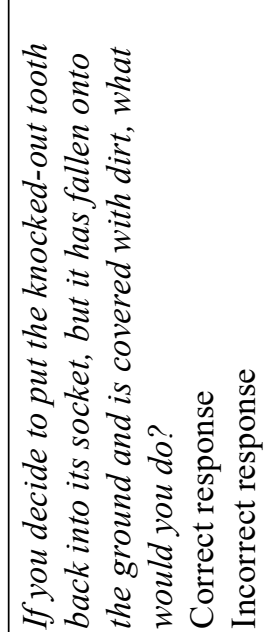 & 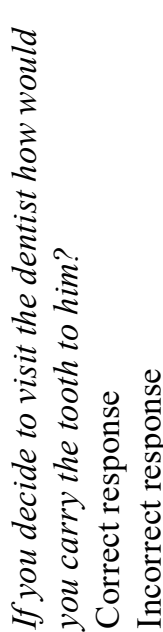 & 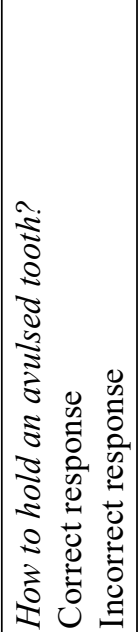 & 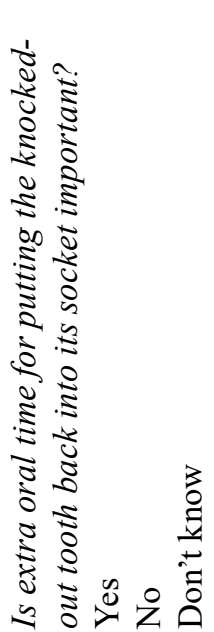 & 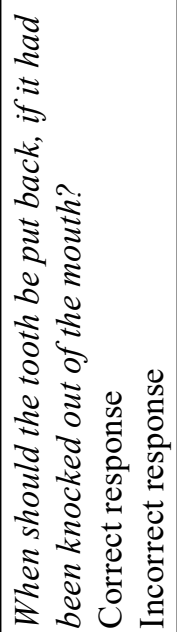 & 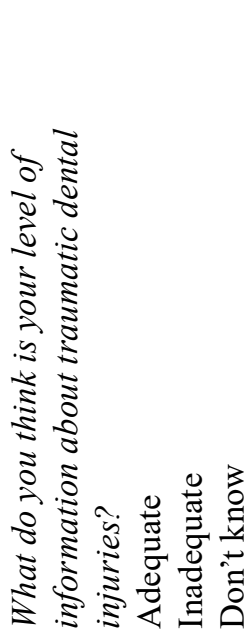 \\
\hline
\end{tabular}




\begin{tabular}{|c|c|c|c|c|c|c|c|c|}
\hline & $x 0$ & $\begin{array}{ll}n & \overline{8} \\
\dot{a} & \vdots \\
0 & 0\end{array}$ & & 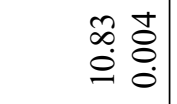 & $\begin{array}{ll}\infty & \overline{8} \\
\stackrel{\sim}{\sim} & 0 \\
0\end{array}$ & $\begin{array}{c}\tilde{n} \\
\stackrel{n}{0}\end{array}$ & $\begin{array}{l}\stackrel{n}{0} \\
\stackrel{n}{+} \frac{0}{0}\end{array}$ & $\begin{array}{ll}\infty & \bar{n} \\
\stackrel{n}{i} & \stackrel{0}{0}\end{array}$ \\
\hline \multirow{3}{*}{$\begin{array}{l}3 \\
3\end{array}$} & 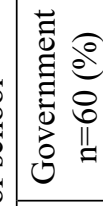 & 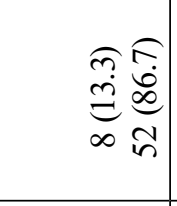 & $\begin{array}{l}\stackrel{\ominus}{\varrho} \\
\stackrel{0}{\varrho} \\
\stackrel{0}{0}\end{array}$ & 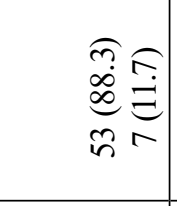 & 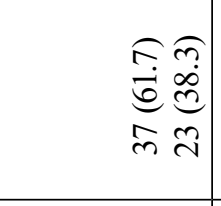 & 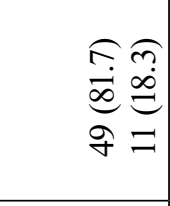 & $\begin{array}{l}\stackrel{\theta}{\varrho} \\
\stackrel{\varrho}{0} \\
\stackrel{0}{0}\end{array}$ & 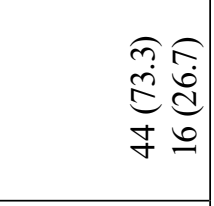 \\
\hline & 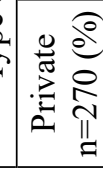 & 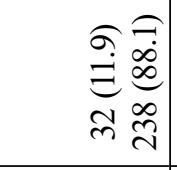 & 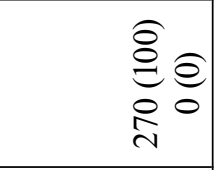 & 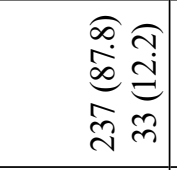 & 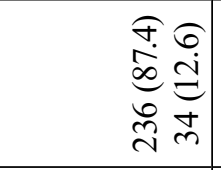 & 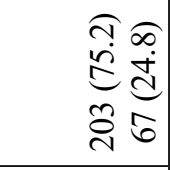 & 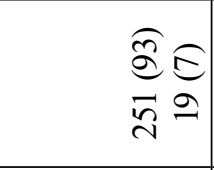 & 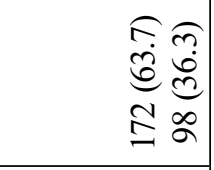 \\
\hline & $x a$ & 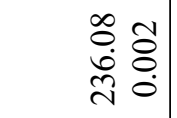 & & $\begin{array}{ll}\curvearrowleft & 0 \\
0 & \overrightarrow{8} \\
& 0\end{array}$ & 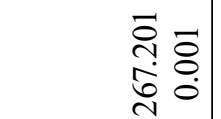 & $\begin{array}{l}\vec{b} \vec{\sigma} \\
\dot{\Xi} \\
=\end{array}$ & $\begin{array}{cc}\vec{r} & \overline{8} \\
\infty \\
\infty & 0 \\
0\end{array}$ & 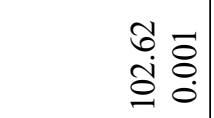 \\
\hline \multirow{4}{*}{ 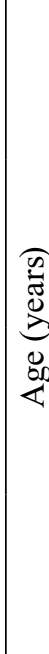 } & 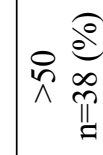 & 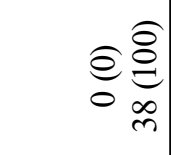 & 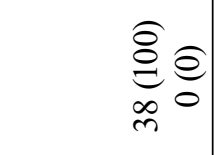 & 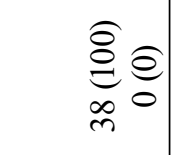 & 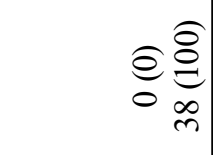 & 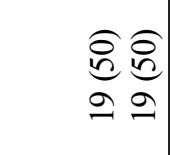 & $\underset{\infty}{\stackrel{\ominus}{\ominus}} \underset{0}{\stackrel{\theta}{e}}$ & 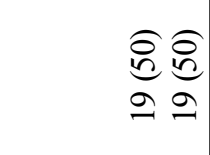 \\
\hline & 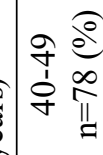 & 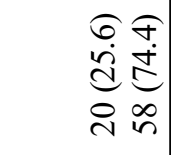 & $\underset{\infty}{\stackrel{\theta}{\ominus}} \underset{0}{e}$ & 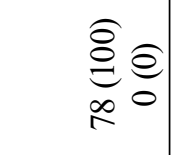 & 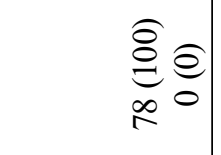 & $\underset{\infty}{\stackrel{\Xi}{\sigma}} \underset{0}{\varrho} \underset{0}{e}$ & 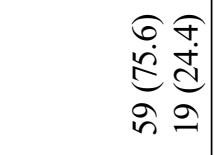 & $\underset{\infty}{\stackrel{\varrho}{\ominus}} \underset{0}{e}$ \\
\hline & के & 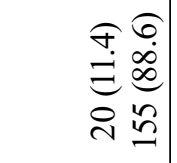 & 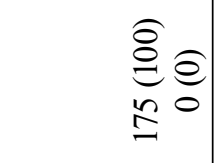 & 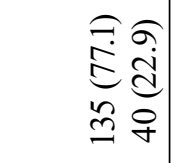 & 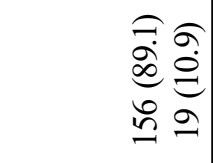 & 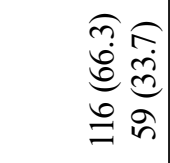 & 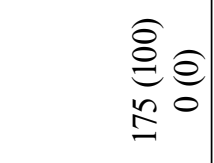 & 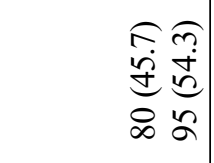 \\
\hline & 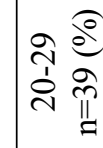 & 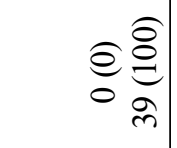 & 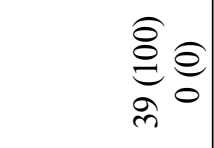 & 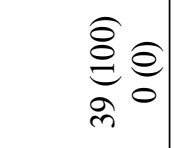 & 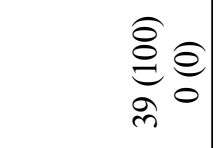 & 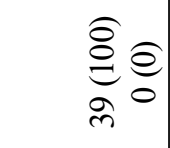 & 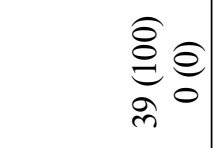 & 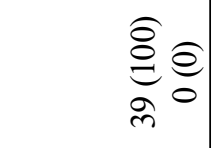 \\
\hline \multicolumn{2}{|r|}{$x 0$} & $\begin{array}{ll}l & \bar{\sigma} \\
& 0 \\
0\end{array}$ & & $\begin{array}{ll}n & \bar{a} \\
\dot{q} & \stackrel{9}{0}\end{array}$ & \begin{tabular}{ll}
0 & $\overline{8}$ \\
\hdashline & 0 \\
0 & 0
\end{tabular} & $\begin{array}{c}\text { mे } \\
= \\
= \\
0\end{array}$ & $\begin{array}{l}\vec{m} \\
\stackrel{\tilde{\sigma}}{\omega} \\
0\end{array}$ & 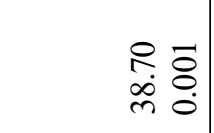 \\
\hline \multirow{2}{*}{ 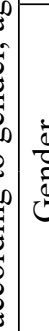 } & 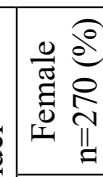 & 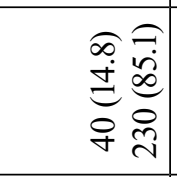 & 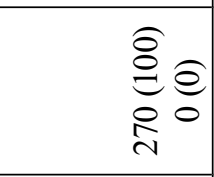 & 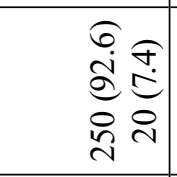 & 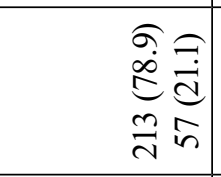 & 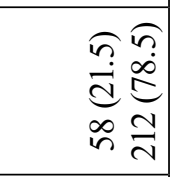 & 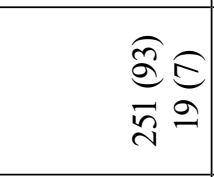 & 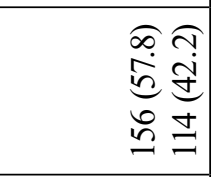 \\
\hline & 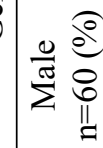 & 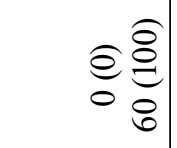 & 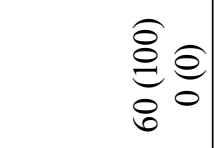 & 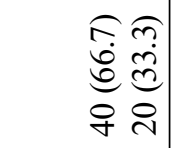 & 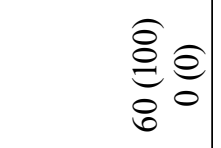 & 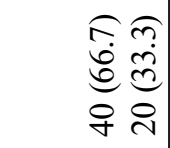 & 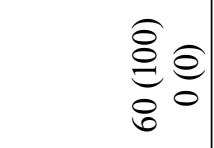 & 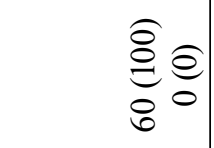 \\
\hline \multicolumn{2}{|r|}{$\begin{array}{l}\stackrel{0}{0} \\
\stackrel{0}{0} \\
\stackrel{0}{0} \\
\tilde{0}\end{array}$} & 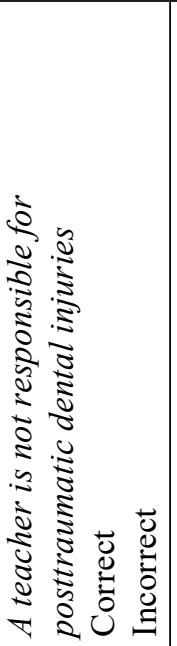 & 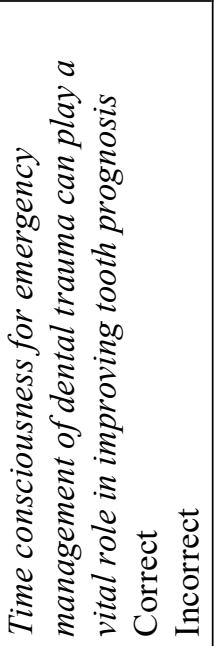 & 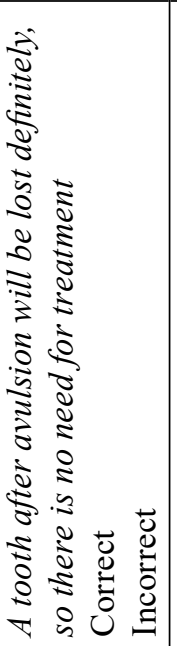 & 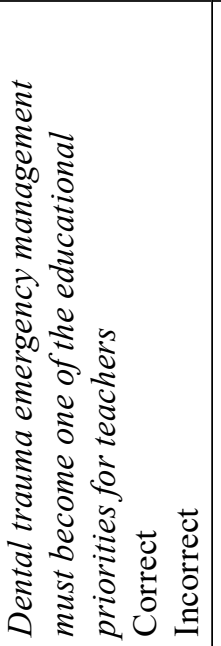 & 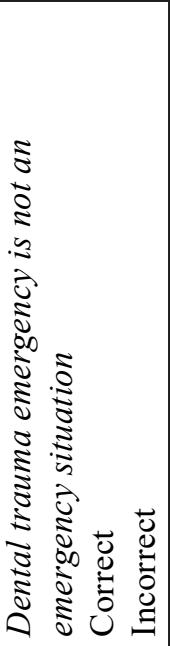 & 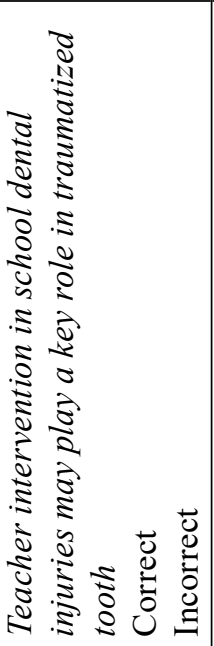 & 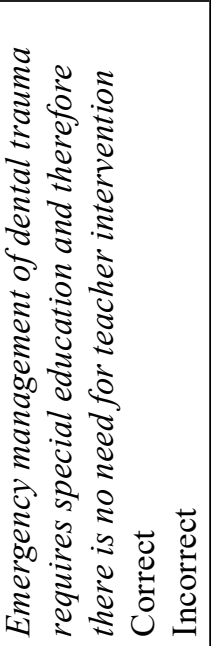 \\
\hline
\end{tabular}




\begin{tabular}{|c|c|c|c|c|}
\hline 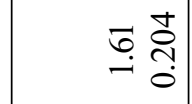 & $\begin{array}{ll}\infty & n \\
0 & 0 \\
0 & 0 \\
0 & 0\end{array}$ & $\stackrel{a}{a} \frac{n}{0}$ & 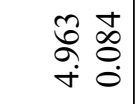 & \\
\hline 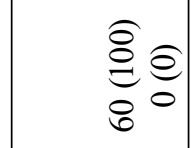 & 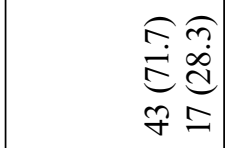 & 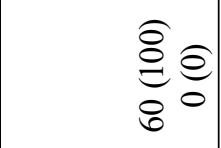 & 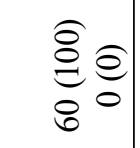 & $\underset{8}{\stackrel{\varrho}{\varrho}} \stackrel{0}{\circ}$ \\
\hline 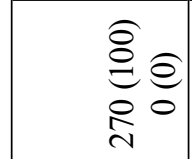 & 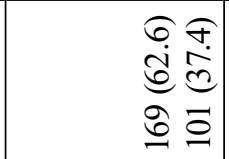 & 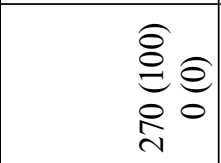 & 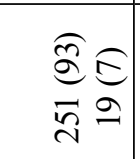 & 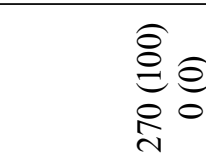 \\
\hline $\begin{array}{l}\tilde{N} \overrightarrow{8} \\
\underset{\sim}{\sim}\end{array}$ & 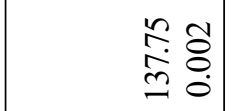 & $\begin{array}{lc}\infty & 0 \\
\infty & \vdots \\
\infty & 0\end{array}$ & $\begin{array}{l}\hat{\sigma} \dot{8} \\
\text { ๙் }\end{array}$ & \\
\hline 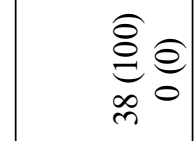 & 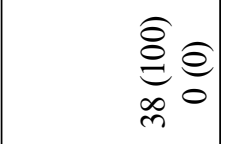 & 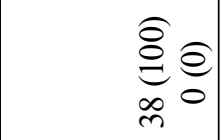 & 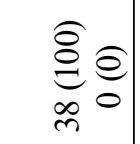 & $\underset{\substack{\infty \\
\stackrel{\varrho}{\varrho}}}{\varrho}$ \\
\hline 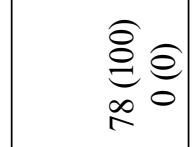 & 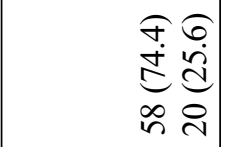 & 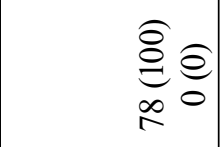 & 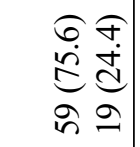 & 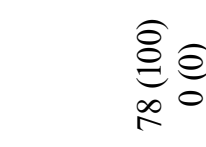 \\
\hline 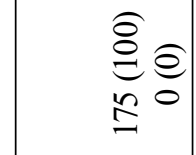 & 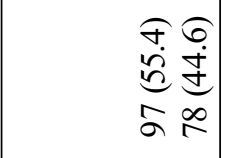 & $\begin{array}{l}\underset{\varrho}{\varrho} \\
\stackrel{\varrho}{=}\end{array}$ & 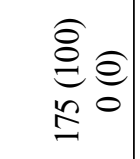 & 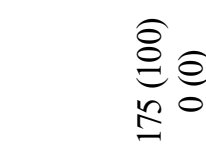 \\
\hline 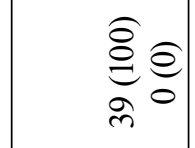 & 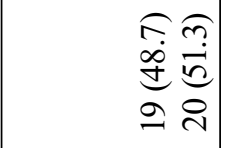 & 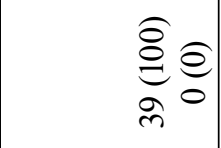 & 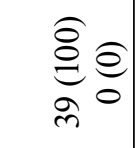 & 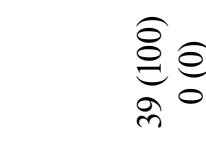 \\
\hline $\begin{array}{l}\bar{n} \\
\\
\dot{0}\end{array}$ & 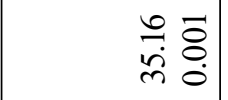 & $\begin{array}{ll}\stackrel{0}{0} \\
\stackrel{\theta}{+} \\
0\end{array}$ & $\begin{array}{cc}\circ & 0 \\
& 0 \\
& 0\end{array}$ & \\
\hline 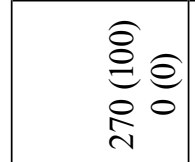 & 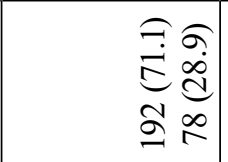 & 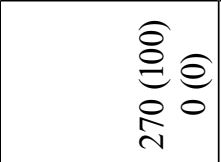 & 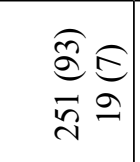 & 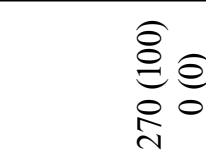 \\
\hline 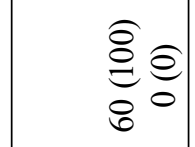 & 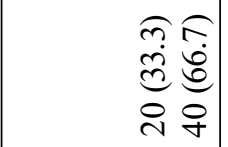 & 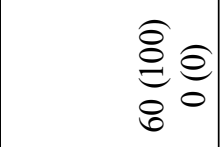 & 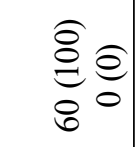 & 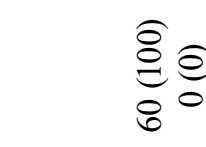 \\
\hline 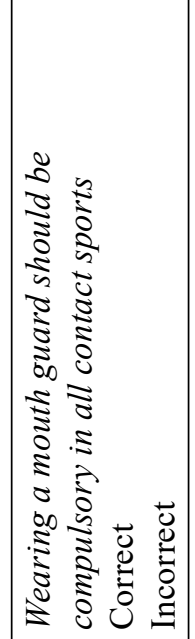 & 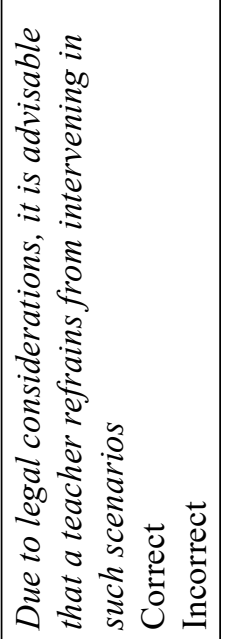 & 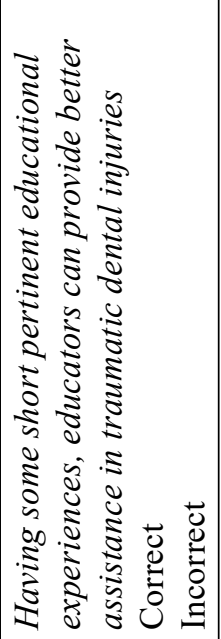 & 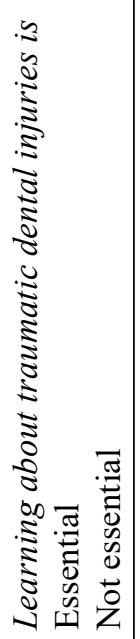 & 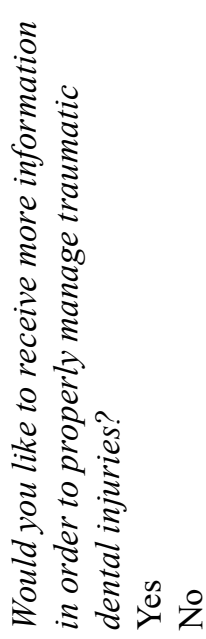 \\
\hline
\end{tabular}




\begin{tabular}{|c|c|c|c|c|c|c|c|c|}
\hline & $x=$ & $\hat{0} \begin{array}{c}n \\
0 \\
0\end{array}$ & ' & 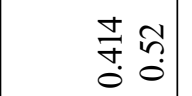 & & 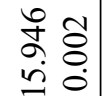 & $\begin{array}{ll}\vec{\infty} & \bar{a} \\
\infty & \bar{\sigma} \\
\vec{\sim} & 0 \\
0\end{array}$ & $\begin{array}{l}\overrightarrow{\widetilde{g}} \tilde{\hat{\sigma}} \\
\stackrel{\gamma}{\dot{\gamma}}\end{array}$ \\
\hline \multirow{2}{*}{$\begin{array}{l}0 \\
0 \\
0 \\
4 \\
0 \\
0 \\
0 \\
0\end{array}$} & 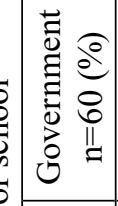 & 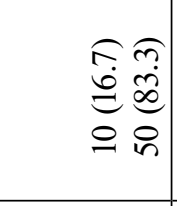 & 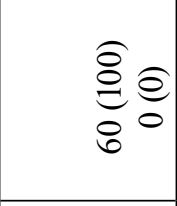 & 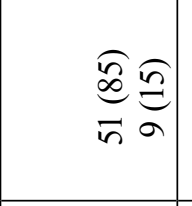 & & 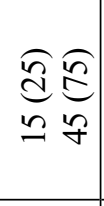 & 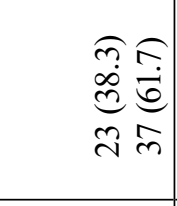 & $\begin{array}{l}\sqrt{\vec{b}} \approx \\
\hat{m} \bar{c}\end{array}$ \\
\hline & 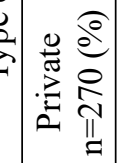 & 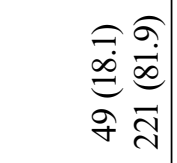 & 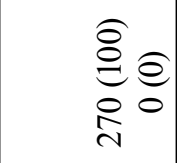 & 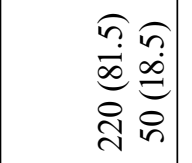 & & 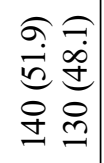 & 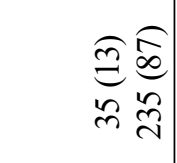 & 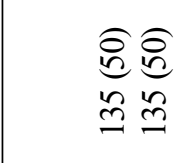 \\
\hline \multicolumn{2}{|r|}{$x=$} & $\begin{array}{ll}\hat{i} & \overline{8} \\
i \\
i n \\
i n\end{array}$ & & $\begin{array}{ll}\vec{b} & \overrightarrow{8} \\
\vec{\nabla} & 0 \\
0\end{array}$ & & 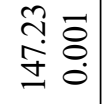 & $\begin{array}{l}\overrightarrow{ } \\
\stackrel{\vec{\Xi}}{\Xi} \\
\dot{0}\end{array}$ & 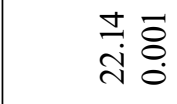 \\
\hline & 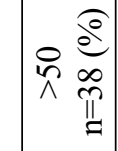 & 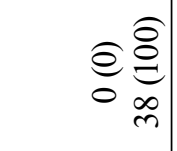 & $\underset{\infty}{\stackrel{\ominus}{e}} \underset{0}{\stackrel{e}{e}}$ & 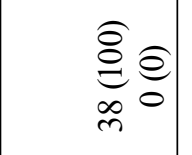 & & $\underset{\substack{\infty \\
\stackrel{\ominus}{\ominus}}}{\stackrel{0}{e}}$ & 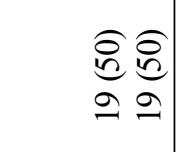 & 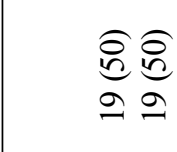 \\
\hline & 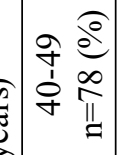 & 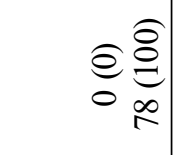 & 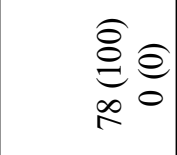 & 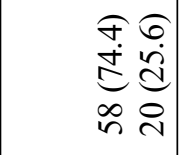 & & 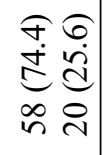 & 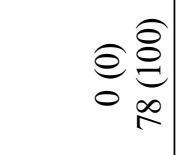 & 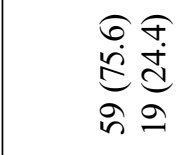 \\
\hline & 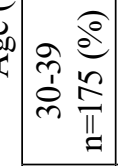 & 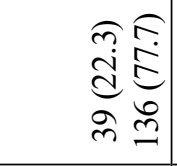 & 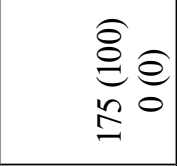 & 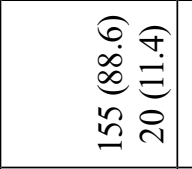 & & 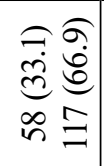 & 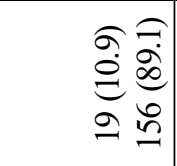 & 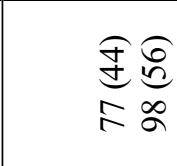 \\
\hline & 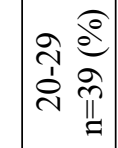 & 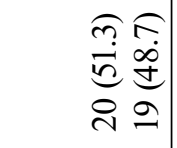 & 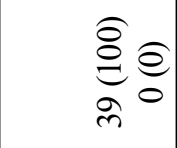 & 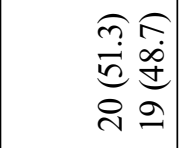 & & 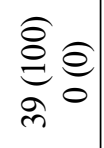 & 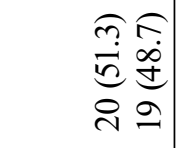 & 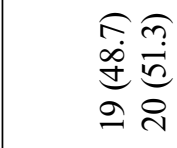 \\
\hline \multicolumn{2}{|r|}{$\approx 0$} & 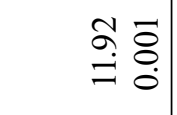 & & $\begin{array}{ll}\sigma & \bar{\sigma} \\
= & 0 \\
\end{array}$ & & $\begin{array}{ll}\infty & \overline{8} \\
\stackrel{0}{0} & 0 \\
& 0\end{array}$ & $\begin{array}{ll}= & \overrightarrow{8} \\
0 & 0 \\
- & 0\end{array}$ & $\begin{array}{l}5 \\
\\
=\end{array}$ \\
\hline \multirow{2}{*}{ 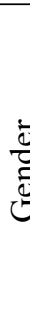 } & 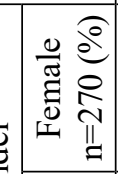 & 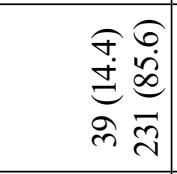 & 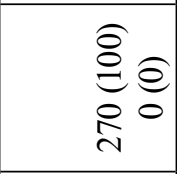 & 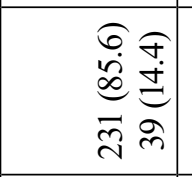 & & 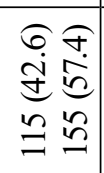 & 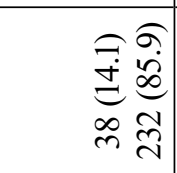 & 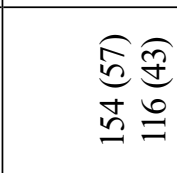 \\
\hline & 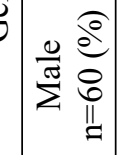 & 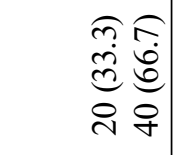 & 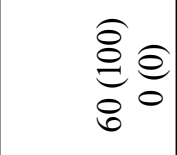 & 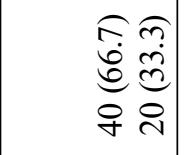 & & 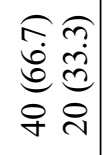 & 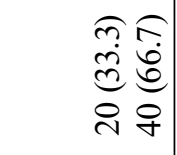 & 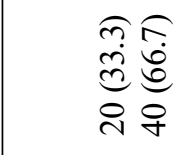 \\
\hline & $\begin{array}{l}\stackrel{0}{0} \\
\stackrel{\tilde{D}}{0} \\
\stackrel{0}{0}\end{array}$ & 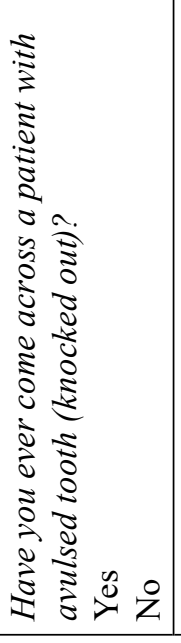 & 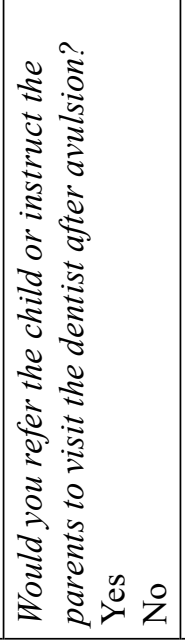 & 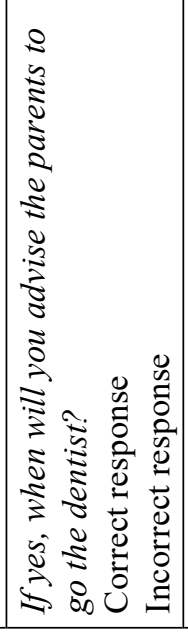 & \multicolumn{2}{|c|}{ 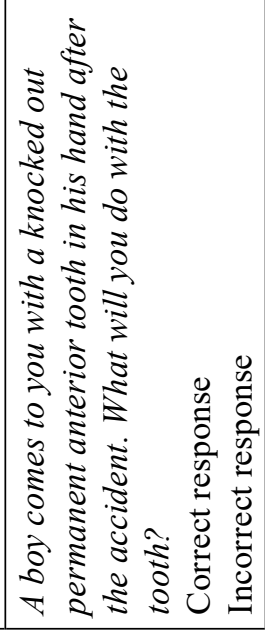 } & 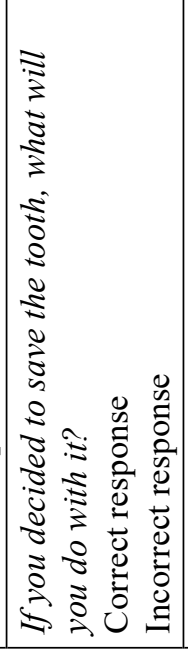 & 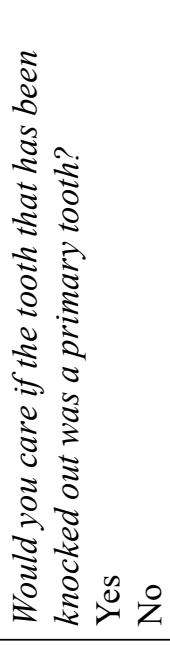 \\
\hline
\end{tabular}




\section{DISCUSSION}

Dental trauma is injury to mouth, including teeth, lips, gums, tongue, and jawbones. Traumatic dental injuries (TDIs) may include knocked-out teeth (dental avulsion), cracked or craze (fractured), displaced teeth (dental luxation, lateral displacement, or extrusion), pushed up into the jawbone (dental intrusion), or loosened teeth (subluxation or dental concussion). TDIs leading to avulsion can be defined as complete displacement of tooth out of socket [8]. Among all types of TDIs, avulsion is the most complicated. Avulsion of permanent teeth is seen in $0.53 \%$ of all dental injuries. Several studies have demonstrated that this injury is one of the most thoughtful dental injuries and the prognosis is very much dependent on the actions taken at the place of accident and promptly after the avulsion and hence, we decided to assess the knowledge of management of avulsion among school teachers.

The major source of information reported was internet which is readily accessible to everyone through the development of technology. Similar findings of internet for young people and television for elderly people were observed by Al-Sane et al. [7]. Recently, Al-Musawi et al. [5] established the provision of information on emergency management of TDI through the use of smartphones.

The current study revealed insufficient proportion of knowledge about avulsion of tooth which can be comparable to previous similar studies. Around half the teachers think that it is necessary to save the avulsed tooth which is in accordance with the similar study conducted by Mohandas and Chandan [19] at Bangalore (49.6\%). This shows the positive attitude of teachers in Bhubaneswar towards the necessity of saving avulsed tooth.

The transport media also play an important role. The most popular choice of storage medium during transportation was antiseptic solution $72.6 \%$. Storing the tooth in an antiseptic solution is not recommended because it will compromise the periodontal ligament cells and thus adversely affect the prognosis of replanted tooth. Many teachers were not aware that dry storage during transportation would seriously prejudice survival of the replanted tooth; the prognosis being dependent on avoidance of injury to periodontal membrane during the time the tooth is out of its socket [29]. The perfect storage medium should be proficient of preserving cell vitality, adherence, and clonogenic capacity and must be readily accessible at the site of accident or easily reachable [9]. Milk has a favourable osmolarity and composition for maintaining the viability of periodontal ligament cells and has been recommended for temporary storage of avulsed teeth before replantation. In addition to being readily available, it preserves cell viability for up to 3 hours [27]. In the present study most of the teachers were unaware of the right method in transporting the affected tooth to dentist which is almost similar to the study conducted by Shamarao et al., [24]. Contrary to this, studies conducted by Singh et al., [26]; Mohandas and Chandan [19]; Ahluwalia et al., [2]; Alluqmani and Omar [4]; Chandukutty et al. [11] and Taranath et al. [27] found that a good number of school teachers, i.e. $87 \%, 49.6 \%, 47.4 \%, 45.3 \%, 40 \%$, and $35.7 \%$, respectively, were aware of correct storage medium.

It was surprising to notice that school teachers of Bhubaneswar were not aware of the importance of extra oral time in managing a dental injury. This was evident from their response regarding the urgency of seeking professional help, where a vast majority were not in favor of seeking immediate professional help. Opposing results were obtained from studies conducted by Chandukutty et al. [11], Taranath et al., [27], Al-Obaida [6] and Kaul et al. [15] as 45.9\%, $33.6 \% .50 \%$ and $78.3 \%$ respectively.

In our study, it was noteworthy to witness that majority of the participants (mostly male teachers) were having better knowledge which reflected in their positive attitude and practice as compared to the female counterparts regarding the need for treatment of loss of avulsed tooth. Contrary results were obtained in other studies conducted by de Lima Ludgero et al. [12] at Brazil (60.4\%) and Hashim [14] at the UAE (44.1\%). This can be owed to caring nature and closeness of females to children.

Responses based on age has shown that a greater number of right answers were provided by younger age population and were also found to practice better methods. This might be because the younger age group is more connected with various sources of information like technology and mass media. Similarly, though statistically not significant the government school teachers demonstrated better awareness which again may be related to improved knowledge in them.

Larger population perceived that their level of knowledge was not satisfactory as they responded for inadequate and don't know options which was similar to other studies $[10,18]$. This indicates the need of organizing educational promotive and preventive programs on dental trauma. Statistically significant $(p<0.05)$ observation was found across all groups wherein they felt the emergency management and their timely intervention towards dental injuries was essential.

When teachers were asked if they would like to receive more information to manage such cases, everyone $(100 \%)$ answered 'yes' and almost similar responses were obtained in studies conducted by Mohandas and Chandan (96.5\%) [19], Olatossio et al. (93.4\%) [20], Chan (81.9\%) [10] and Uppal et al., (76.4\%) [30]. 
In our study, less than half the teachers have come across the cases of avulsed tooth. Such situations were responded to be managed by referring to nearby dentists and majority were aware of the immediate actions to be taken without wasting time which is analogous with the study conducted in Saudi [6]. In contrast majority of the teachers would call the parent and ask them to take the child to a dentist, while one-third were not able to decide on what to do under such circumstances [23]. It was appreciable to observe that at least half the subjects would care even if the avulsed tooth was a primary tooth. Early loss of a primary tooth may affect the physiological sequence of permanent teeth and may be an etiological factor for malocclusion.

Reports show that more than half of all children suffer traumatic dental injuries in school $[16,25]$ and this highlights the importance of experienced/ trained school staff, who are often required to respond initially to traumatic event. Instantaneous response to dental injury can reduce the level of children's and their parents' apprehension with respect to succeeding dental treatment [17]. Therefore, teachers who work with children must be aware of the importance of emergency treatment and of how to proceed in cases of tooth avulsion. Educational programs and dental camps can prove to be beneficial in this regard.

\section{LIMITATIONS}

As participants' responses were self-reported it may be considered as a limitation and caution must be exercised with generalization of these findings. It's also advisable to include teachers from all the schools in Bhubaneswar and consider many more variables.

\section{CONCLUSION}

This study has demonstrated the lack of knowledge among school teachers regarding correct handling, transportation, and storage of an avulsed tooth and stressed the immediate need for dental health education to be stepped up among school teachers. By increasing knowledge on how avulsed teeth are to be dealt with at the site of the accident, the risk of permanent tooth loss is minimized. The prognosis of an avulsed tooth is good if it is replanted under ideal circumstances, and the tooth can often be retained for life.

\section{Acknowledgements}

The authors would like to thank the study participants for their kind cooperation in the study.

\section{Disclosures}

The authors declared no potential conflicts of interest with respect to the authorship and/or publication of this article.

\section{Funding source}

This research did not receive any specific grant from funding agencies in the public, commercial, or notfor-profit sectors.

\section{REFERENCES}

1. Abanto J., Tello G., Bonini G.C., Oliveira L.B., Murakami C., Bonecker M.: Impact of traumatic dental injuries and malocclusions on quality of life of preschool children: a population-based study. Int J Paediatr Dent. 2014;25:18-28.

2. Ahluwalia P., Pannu P., Kalra S., Kaur A., Behl D., Gambhir R.S.: Assessment of knowledge and attitudes of school teachers regarding emergency management of an avulsed permanent tooth. Saint Int Dent J. 2015;1:16-21.

3. Aldrigui J.M., Jabbar N.S., Bonecker M., Braga M.M., Wanderley M.T.: Trends and associated factors in prevalence of dental trauma in Latin America and Caribbean: a systematic review and meta-analysis. Community Dent Oral Epidemiol. 2014;42:30-42.

4. Alluqmani F.A., Omar O.M.: Assessment of school teachers' knowledge about management of traumatic dental injuries in Al-Madinah city, Saudi Arabia. Eur J Dent. 2018;12:171-175.

5. Al-Musawi A., Al-Sane M., Andersson L.: Smartphone App as an aid in the emergency management of avulsed teeth. Dent Traumatol. 2017;33:13-18.

6. Al-Obaida M.: Knowledge and management of traumatic dental injuries in a group of Saudi primary school's teachers. Dent Traumatol. 2010.;26:338-341.

7. Al-Sane M., Bourisly N., Almulla T., Andersson L.: Laypeoples' preferred sources of health information on the emergency management of tooth avulsion. Dent Traumatol. 2011;27:432-437.

8. Andreasen J.O., Andreasen F.M., Andersson L.: Textbook and Color Atlas of Traumatic Injuries to the Teeth. Wiley Blackwell: John Wiley \& Sons. 2013;383425.

9. Ashkenazi M., Marouni M., Sarnat H.: In vitro viability, mitogenicity and clonogenic capacity of periodontal ligament cells after storage in four media at room temperature. Endod Dent Traumatol. 2000;16:63-70.

10. Chan A.W., Wong T.K., Cheung G.S.: Lay knowledge of physical education teachers about the emergency management of dental trauma in Hong Kong. Dent Traumatol. 2001;17:77-85.

11. Chandukutty D., Peedikayil F.C., Premkumar C.T., Narasimhan D., Jose D.: Awareness of dental trauma management among school teachers of Kannur, Kerala, India. J Clin Diagn Res. 2017;11:ZC08-12.

12.de Lima Ludgero A., de Santana Santos T., Fernandes A.V., de Melo D.G., Peixoto A.C., da Costa Araujo 
F.A., Dourado A.T., Gomes A.C.A.: Knowledge regarding emergency management of avulsed teeth among elementary school teachers in Jaboatão dos Guararapes, Pernambuco, Brazil. Indian J Dent Res. 2012;23:585-590.

13. Glendor $U .:$ Epidemiology of traumatic dental injuries - a 12 year review of the literature. Dent Traumatol. 2008;4:603-611.

14. Hashim R.: Dental trauma management awareness among primary school teachers in the Emirate of Ajman, United Arab Emirates. Eur J Paediatr Dent. 2011;12:99-102.

15. Kaul R., Jain P., Saha N., Goswami S., Mukhopadhyay S., Saha S., Sarkar S.: Evaluation of knowledge, awareness, and attitude toward emergency dental trauma management among the school teachers of Kolkata. Indian J Dent Res. 2017;28:595-603.

16. Kaur H., Kaur S., Kaur H.: Prehospital emergency management of avulsed permanent teeth: Knowledge and attitude of school teachers. Indian $\mathrm{J}$ Dent Res. 2012;23:556-561.

17. Levin L., Friedlander L.D., Geiger S.B.: Dental and oral trauma and mouthguard use during sport activities in Israel. Dent Traumatol. 2003;19:237-242.

18. McIntyre J.D., Lee J.Y., Trope M., Vann W.F.: Elementary school staff knowledge about dental injuries. Dent Traumatol. 2008;24:289-298.

19. Mohandas U., Chandan G.D.: Knowledge, attitude and practice in emergency management of dental injury among physical education teachers: A survey in Bangalore urban schools. J Indian Soc Pedod Prev Dent. 2009;27:242-248.

20.Olatosi O.O., Iwuala S.O., Isiekwe G.I., Oredugba F.A., Adenaike A.S., Oluwo A.O.: Knowledge and attitude of some Nigerian school teachers on the emergency management of avulsed permanent incisor. J West Afr Coll Surg. 2013;3:30-52.

21. Porritt J.M., Rodd H.D., Baker S.R.: Quality of life impacts following childhood dento-alveolar trauma. Dent Traumatol. 2011;27:2-9.

22.Pourat N., Nicholson G.: Unaffordable dental care is linked to frequent school absences. Policy Brief UCLA Cent Health Policy Res. 2009:1-6.
23.Pujita C., Nuvvula S., Shilpa G., Nirmala S., Yamini V.: Informative promotional outcome on school teachers' knowledge about emergency management of dental trauma. J Conserv Dent. 2013;16:21-27.

24. Shamarao S., Jain J., Ajagannanavar S.L., Haridas R., Tikare S., Kalappa A.A.: Knowledge and attitude regarding management of tooth avulsion injuries among school teachers in rural India. J Int Soc Prev Community Dent. 2014;4 Suppl 1:S44-48.

25.Shashikiran N.D., Reddy V.V., Nagaveni N.B.: Knowledge and attitude of 2,000 parents (urban and rural - 1,000 each) with regard to avulsed permanent incisors and their emergency management, in and around Davangere. J Indian Soc Pedod Prev Dent. 2006;24:116-121.

26. Singh M., Ingle N.A., Kaur N., Yadav P.: Evaluation of knowledge and attitude of school teachers about emergency management of traumatic dental injury. J Int Soc Prev Community Dent. 2015;5:108-113.

27. Taranath M., Senaikarasi R.M., Manchanda K.: Assessment of knowledge and attitude before and after a health education program in East Madurai primary school teachers with regard to emergency management of avulsed teeth. J Indian Soc Pedod Prev Dent. 2017;35:63-67.

28. Tello G., Bonini G.C., Murakami C., Abanto J., Oliveira L.B., Bonecker M.: Trends in the prevalence of traumatic crown injuries and associated factors in Brazilian preschool children: 10-year observational data. Dent Traumatol. 2016;32:274-280.

29. Trope M.: Clinical management of the avulsed tooth. Dent Clin North Am. 1995;39:93-112.

30. Uppal A., Thakur S., Singhal P., Chauhan D., Jayam C., Doneria D.: Knowledge, Attitude and Practice (KAP) of Teachers Regarding Dental Traumatic Injuries Among School Children of Shimla City. Int J Recent Sci Res. 2016;7:11236-11241.

Received: 04.04 .2020

Accepted: 28.06 .2020

This article is available in Open Access model and licensed under a Creative Commons Attribution-Non Commercial 3.0.Poland License (CC-BY-NC) available at: http://creativecommons.org/licenses/by-nc/3.0/pl/deed.en 